Zuständigkeit des Oberlandesgerichts die Übernahme der Verfolgung durch den

GBA ist! Das ist auch nicht mehr im Wege einer verfassungskonformen Auslegung korrigierbar, denn kein Gericht kann eine Staatsanwaltschaft zur Übernahme einer Strafsache verpflichten - \iso GVG.

Bei den prozessual späteren Entscheidungen gem. $\iint 225 a, 270$ StPO ergibt sich das gleiche.

\title{
Uwe Günther/Dietz Tretschok Vom Strahlenschutz zur Informationsherrschaft über Strahlen - Das Strahlenschutzvorsorgegesetz 1986
}

\section{Inhalt und Bedeutung des Strablenschutzvorsorgegesetzes}

Ein Dreivierteljahr nach der Katastrophe von Tschernobyl ist die massive Angst der Bevölkerung über radioaktive Umweltbelastung alltäglichem Gleichmut gewichen. So wird die Cäsiumbelastung der Milch ebenso akzeptiert wie deren Verfallsdatum. Die langfristigen Folgen der Katastrophe von Tschernobyl sind als gesellschaftliches Thema nicht aktuell. Bleibend geändert hat sich jedoch das juristische Verhältnis zum Gefährdungspotential atomarer Anlagen. Hierfür steht das Strahlenschutzvorsorgegesetz (StrVG). War vor Tschernobyl versucht worden, das Gefährdungspotential atomarer Anlagen mit Begriffen wie »Restrisiko«, das nach den Kriterien der "praktischen Vernunft« beurteilt werden sollte, auszuschließen, so ist nun ein Handlungsrahmen geschaffen worden, der bei dem jetzt durchaus auch praktisch denkbaren GAU das Restrisiko beherrschbar und die Folgen handhabbar machen soll.

Durch das Strahlenschutzvorsorgegesetz sollen »die rechtlichen Grundlagen für ein effektives und koordiniertes Vorgehen aller beteiligten Dienststellen in Bund und Ländern geschaffen werden " $^{1}$. Zweck des Gesetzes ist, unter Beachtung des Standes der Wissenschaft und unter Berücksichtigung aller Umstände durch angemessene Maßnahmen die Strahlenexpositionen des Menschen und die radioaktive Kontamination der Umwelt so gering wie möglich zu halten ( $\$$ I Satz 2 StrVG). Um dies zu erreichen, ermitteln Bund und Länder die erforderlichen Daten ( $\$ \$ 2$ und 3 StrVG), die in ein Informationssystem des Bundes einfließen $(\$ 4$ StrVG). Der Bundesminister für Umwelt, Naturschutz und Reaktorsicherheit bewertet die gesammelten Daten $(\$ s$ StrVG). Er ist auch ermächtigt, durch Rechtsverordnungen Dosiswerte, Kontaminationswerte und Berechnungsverfahren festzulegen ( $\$ 6 \mathrm{StrVG}$ ) und Verbote und Beschränkungen bei Lebensmitteln, Futtermitteln, Arzneimitteln und sonstigen Stoffen zu erlassen ( $\$ 7$ StrVG) sowie Empfehlungen an die Bevölkerung auszusprechen $(\$ 9$ StrVG). An den Grenzen können die zur Einhaltung der Kontaminationswerte nach $₫ 6$ StrVG und zur Überwachung der Verbote nach $₫ 7$ StrVG erforderlichen Maßnahmen getroffen werden ( $\$ 8$ StrVG). Die Durchführung des Gesetzes erfolgt im Auftrag des Bundes und in bundeseigener Verwaltung.

I BT-Drucks. 10/6639, S. I. 
Für das Ermitteln, Übermitteln, Zusammenfassen, Aufbereiten und Dokumentieren von Daten über Radioaktivität werden allgemeine Verwaltungsvorschriften erlassen ( $($ Io). Soweit die wesentlichen Punkte des Gesetzes.

Wie sähe ein Szenario bei einem "Ereignis mit nicht unerheblichen radiologischen Auswirkungen « aus? Bundesweit wird gemessen, die Länder übermitteln die Daten, die beim Bundesminister für Umwelt, Naturschutz und Reaktorsicherheit zusammenlaufen. Ihm obliegt die Bewertung der Daten, die er in Zusammenarbeit mit der Strahlenschutzkommission vornimmt. Im Eilverfahren kann er ohne Mitwirkung der Bundesländer Rechtsverordnungen erlassen, die die zulässigen Dosiswerte, Kontaminationswerte und Berechnungsverfahren festlegen. Sind entsprechende Verordnungen bereits vorher erlassen worden, kann er im Eilverfahren die Werte an die jeweilige Situation anpassen. Ein Minimierungsgebot existiert nicht. Gemäß $₫$ I Nr. 2 StrVG sind die Maßnahmen lediglich unter Berücksichtigung aller Umstände zu treffen. Mit anderen Worten: Je größer die Katastrophe, desto höher die zugelassenen Werte. Bei der Festlegung der zulässigen Werte und der damit verbundenen Einschätzungen hat der Bundesminister für Umwelt, Naturschutz und Reaktorsicherheit exklusive Kompetenz: Die Länder können auch dann keine niedrigeren Werte festsetzen, wenn regional unterschiedliche Bestrahlung festzustellen ist. Freie Meßstellen wie etwa unabhängige Institute sind aus dem System ausgekoppelt und gelten nicht als qualifiziert. Der Bundesminister kann des weiteren Empfehlungen an die Bevölkerung aussprechen. Dies zwar im Benehmen mit den Bundesländern, letztlich dürfte es jedoch auch insoweit zu bundeseinheitlichen, vom Bundesminister ausgesprochenen Empfehlungen kommen, weil regionale Empfehlungen der Intention des Gesetzes, Verunsicherungen der Bevölkerung zu vermeiden, zuwiderlaufen würden.

An die Werte und Empfehlungen sind Entschädigungszahlungen gemäß $\ 38$ des Atomgesetzes gebunden. Insofern ist es nicht abwegig zu vermuten, daß auch finanzielle Interessen des Bundes bei der Festsetzung der Werte und der Form der Empfehlungen eine Rolle spielen werden. Dies insbesondere dann, wenn das »Ereignis « im Ausland passiert und dort höhere Grenzwerte als in der Bundesrepublik Deutschland gelten.

Mit dem Gesetz wird erreicht, daß bundesweit nur noch vom Bundesminister für Umwelt, Naturschutz und Reaktorsicherheit genehmigte Daten verbreitet werden können. Unterschiedliche Regelungen in den Ländern wird es nicht mehr geben. Der Bundesminister bestimmt den Grad der Gefährdung der Bevölkerung; er ist befugt zu entscheiden, welche Konsequenzen zu ziehen und welche Maßnahmen zu treffen sind.

\section{Das Gesetzgebungsverfabren}

Am II.7. 1986 hatte der Bundesrat einer Entschließung zugestimmt, in der die Bundesregierung aufgefordert wurde, unter Beteiligung der Länder »Rechtsgrundlagen und organisatorische Maßnahmen zur Bewältigung länderübergreifender radioaktiver Belastungen « zu schaffen. ${ }^{2}$ Am 3.9. 1986 stellte die Bundesregierung ein Arbeitsprogramm vor, in dem es unter anderem heißt: "Die Bundesregierung hat die Sorgen der Bevölkerung im Zusammenhang mit der großflächigen Kontamination von Lebens- und Futtermitteln sehr ernst genommen und wird sich nachdrück-

2 Vgl. A.Clauss, Begründung der Ablehnung des StrVG vor dem Bundesrat, FR. v. 27. I I. 86, S. 10. 
lich dafür einsetzen, daß zukünftig unterschiedliche Empfehlungen (!), die die

Bevölkerung verunsichern können, vermieden werden. « Am 18.9. 1986 wurde der "Gesetzentwurf zur Überwachung der Radioaktivität in der Umwelt (Umweltradioaktivitätsgesetz) * veröffentlicht. Dieser Gesetzentwurf enthielt im wesentlichen die Konzeption des jetzigen Strahlenschutzvorsorgegesetzes. Am 29.9. 1986 legte die Regierung nach sprachlichen Korrekturen und inhaltlichen Verfeinerungen das Strahlenschutzvorsorgegesetz vor, das am 2. 10. 1986 in I. Lesung behandelt und an die Ausschüsse überwiesen wurde.

Den Sachverständigen wurde eine Frist bis zum 30. 10. 1986 zur Beantwortung von Fragen eingeräumt. Dieses Verfahren hatte zur Folge, daß die Ausschußmitglieder die schriftlichen Vorlagen der Sachverständigen teilweise erst während der Anhörung am 3. I I. I 986 zu Gesicht bekamen. Infolge der Kurzfristigkeit des Verfahrens erschienen einige geladene Sachverständige nicht oder waren nicht bereit, unter einem derartigen zeitlichen Druck wissenschaftlich fundierte Aussagen zu machen. ${ }^{3}$ Am I 2. I I. I 986 legten die Koalitionsfraktionen Änderungsanträge als Ergebnis der Beratungen mit dem Bundesrat als Tischvorlage im Ausschuß vor, wobei diese bereits in das Gesetz eingearbeitet waren. Die Ausschußmitglieder der SPD und der GRÜNEN verließen unter Protest die Sitzung, nachdem ein Antrag auf Verschiebung der Sitzung abgelehnt worden war. Verabschiedet wurde das Gesetz am I I. I 2. 1986, in Kraft ist es seit dem 31. I 2. I986.4

Die Folgen von Tschernobyl werden durch das Gesetz nicht erfaßt, eine neue Katastrophe ist dann, wenn man die Auffassung der Bundesregierung in Sachen Atomenergie teilt, unwahrscheinlich. Erklärbar wird das Verhalten der Bundesregierung, das Gesetz schnell zu verabschieden, nur unter dem Aspekt, daß sie "Handlungsfähigkeit demonstrieren « wollte. Für die Bundesregierung ist nicht die Katastrophe von Tschernobyl das Problem, sondern es sind die unterschiedlichen Empfehlungen, die anläßlich der radioaktiven Belastung der Bundesrepublik Deutschland ausgesprochen wurden. Nicht objektive Gefahrenlagen, sondern deren subjektive Verarbeitung im Bewußtsein der Bevölkerung möchte die Bundesregierung ändern. In diesem Zusammenhang läßt das Verfahren zur Verabschiedung des StrVG das Schlimmste für dessen zukünftige Anwendung vermuten, da der Machtzuwachs des Bundesministers für Umwelt, Naturschutz und Reaktorsicherheit in seinem Informations- und Bewertungsmonopol besteht und zu befürchten ist, daß bei künftigen Katastrophen dieses rechtliche Instrumentarium ausschließlich unter dem Aspekt der Öffentlichkeitswirksamkeit angewendet wird.

\section{Zu einzelnen Fragen}

\section{Das Minimierungsgebot}

Die Koalitionsfraktionen vertreten die Ansicht, daß die Formulierung »unter Berücksichtigung aller Umstände« nur den ohnehin geltenden Grundsatz der Verhältnismäßigkeit wiedergebe; das Minimierungsgebot ergebe sich eindeutig aus den

3 So z. B. Prof. Dr. Scholz, der den Ausschußvorsitzenden daran erınnerte, daß der Bürger vom Gesetzgeber sorgfälıges Nachdenken erwarte und nicht hastıges Handeln (Unterlagen der Anhörung v. 3.1 I. 86 beım Ausschuß für Umwelt, Naturschutz und Reaktorsıcherheıt).

4 Gesetz zum vorsorgenden Schutz der Bevölkerung gegen Strahlenbelastung (Strahlenschutzvorsorgegesetz - StrVG) vom 19. I 2. I 986, BGBl. I S. 26ro. Gemäß $\$$ I 8 StrVG ist es am Tage nach der Verkündung Im Bundesgesetzblatt (das war der 30. 12. 1986) in Kraft getreten. 
Worten "so gering wie möglich zu halten. "s Ob diese Interpretation des Minimierungsgebots identisch mit der ist, die bislang für das Minimierungsgebot galt, ist mehr als fraglich. Das Minimierungsgebot des $\$ 4 s$ der Strahlenschutzverordnung bedeutet nicht nur einfach, daß die Belastung so gering wie möglich zu halten ist, sondern auch, daß die geltenden Werte keine Höchstwerte sind und daß sie nur für die ungünstigsten Einwirkungsstellen unter ungünstigsten Bedingungen erreicht werden dürfen. Demgegenüber wurde während der Anhörung vom 3.11. 1986 deutlich, daß beabsichtigt ist, Untergrenzen einzuführen, wobei sich die Grenzwerte an den Empfehlungen der EG oder der ICRP orientieren sollen. Unterhalb dieser Grenzen soll weder gemessen werden noch dürfen Behörden Empfehlungen aussprechen. Damit ist jedoch das Minimierungsgebot beseitigt. ${ }^{6}$

Die Formulierung "unter Berücksichtigung aller Umstände « läßt Raum für eine Interpretation, die sich nicht ausschließlich an Grundsätzen der Gesundheitsfürsorge orientiert. Eine solche Interpretation dürfte verfassungsrechtlich jedenfalls dann im Hinblick auf Art. 2 GG mehr als problematisch sein, wenn sie einhergeht mit einer drastischen Erhöhung der Grenzwerte.

\section{Berechnungsverfabren}

Grundlage der Berechnung von Grenzwerten wird bei der Anwendung des StrVG das "effektive Dosismodell" sein, nach dem bereits die Strahlenschutzkommission die Werte nach Tschernobyl ermittelt hat. Dies entspricht nach Ansicht der Bundesregierung dem Stand der Wissenschaft. Das »effektive Dosismodell « wurde von der ICRP vorgestellt und ist Bestandteil der EG-Richtlinien, jedoch wissenschaftlich umstritten. Es erfaßt einerseits Risikogruppen der Bevölkerung wie Kleinkinder oder werdendes Leben nicht. Andererseits rechnet es mit dem "Mortalitätsfaktor ", d. h. die Gewichtungsfaktoren werden danach bemessen, wieviele Todesfälle durch Krebserkrankungen bei Bestrahlung einzelner Organe auftreten. Es werden also nur die zum Tode führenden Krebsfälle berücksichtigt. Krebserkrankungen als Maß für die Bewertung des Risikos fallen aus diesem Berechnungsmodell heraus.

\section{Informationsmonopol/Länderbeteiligung}

Der Bundesminister für Umwelt, Naturschutz und Reaktorsicherheit ist nach $\$ 6$ Abs. I Nr. 3 StrVG ermächtigt, die Berechnungsverfahren festzulegen (die nach dem oben skizzierten Modell erfolgen werden). Er entscheidet also darüber, nach welchen Verfahren die radioaktive Belastung der Umwelt und der Bevölkerung ermittelt wird, und er hat letztlich auch die Verfügungsgewalt über die Meßstellen. Damit werden solche Institutionen, die außerhalb dieses Systems Daten ermitteln, von vornherein disqualifiziert, wenn sie sich nicht an die vorgegebenen Berechnungsverfahren halten. Die Verbreitung der Ergebnisse obliegt dem Bundesminister; er allein entscheidet, welche Daten wann und wo veröffentlicht werden. Ein Zugang zum Informationssystem "Radioaktivität in der Umwelt« für die Bevölkerung ist nicht vorgesehen. Den Ländern stehen die Daten zwar zur Verfügung, aber die Handlungsmöglichkeiten sind begrenzt auf "Ereignisse mit ausschließlich örtlichen Wirkungen «; eine nähere Definition dieser Ereignisse erfolgt nicht, so daß völlig unklar ist, ob es solche Ereignisse überhaupt geben kann.

Im Prinzip ist die Rolle der Länder auf die Ermittlung und die Übermittlung von

5 BT-Drucks. 10/6639, S. 19

6 Vgl. Protokoll der Anhörung v. 3. I 1.86 zum StrVG. 
Daten beschränkt. Eine Bewertungskompetenz ist ihnen bis auf das »örtlich begrenzte Ereignis « nicht eingeräumt. Unter dem Aspekt der Effektivität dürfte es der Bundesminister für Umwelt, Naturschutz und Reaktorsicherheit schwer haben, seine Bewertungskompetenz durchzusetzen: Beim derzeitigen Stand politischer Auseinandersetzungen steht nicht zu erwarten, daß die sozialdemokratischen Umweltminister bzw. der Umweltminister der GRÜNEN bei einer erneuten Katastrophe die Bewertungen des Bundesministers schweigend hinnehmen werden. Dies lassen Äußerungen der Minister der Opposition vermuten. Auch wenn diese oder die CDU-Umweltländerminister schweigen wollten, hätten sie es schwer, das Schweigen im Amt durchzuhalten, wenn ihre Bewertung massiv von der Bevölkerung hinterfragt wird.

Unter juristischen Aspekten ist die Bewertungskompetenz ein neues verwaltungsrechtliches Instrument, das eine alte verfassungsrechtliche Streitfrage aktualisiert: Können Personen, die öffentliche Positionen innehaben, infolge ihrer Stellung das Grundrecht auf Meinungsfreiheit verlieren? Diese Streitfrage ist in der Vergangenheit vielfach gerichtlich unter dem Begriff des "politischen Mandats der Studentenschaft« ungerechtfertigt zu Lasten der Meinungsfreiheit entschieden worden. $\mathrm{Ob}$ die Bewertungskompetenz des Bundesministers vor dem Verfassungsgericht Bestand haben wird, ist immerhin zweifelhaft, zumal seine Kompetenz so weit geht, daß die Länderumweltminister selbst dann zum Schweigen verdammt sind, wenn der Bundesminister Empfehlungen ausspricht, die jenseits des Standes der Wissenschaft sind.

\section{Strablenschutzkommission}

Die Strahlenschutzkommission wird nicht ausdrücklich im StrVG erwähnt. Dennoch kommt ihr eine zentrale Rolle bei der Durchsetzung des Gesetzes und bei der Festsetzung der Grenzwerte und der Berechnungsverfahren zu. Die Strahlenschutzkommission hat zwar keine Befugnisse für nach außen verbindliche Entscheidungen, sie ist lediglich ein Beratungsorgan des zuständigen Bundesministers. Bereits in der Vergangenheit zeigte sich jedoch, daß sie das eigentliche Entscheidungsgremium bei der Festsetzung von Grenzwerten war. Deswegen ist zu vermuten, daß die Strahlenschutzkommission auch die Grenzwerte und die Berechnungsverfahren gemäß den Bestimmungen des StrVG festlegt. Für die Strahlenschutzkommission ist das »effektive Dosismodell« der »Stand der Wissenschaft«, und sie spricht sich auch für eine untere Grenze aus, unterhalb derer nicht mehr gemessen werden soll. Faktisch dürfte mithin der Strahlenschutzkommission eine außerordentlich wichtige Bedeutung zukommen. Im StrVG ist sie dennoch nicht erwähnt. Eine gesetzliche Grundlage für ihr Wirken besteht nicht. Sie ist durch eine Bekanntmachung des Bundesministers des Inneren ins Leben gerufen worden. Personell ist sie ausnahmslos mit Befürwortern atomarer Technologien besetzt. Die Strahlenschutzkommission ist - sowohl was ihre rechtliche Grundlage als auch was ihre personelle Zusammensetzung betrifft - verfassungsrechtlich problematisch, wenn man berücksichtigt, daß sie es ist, die maßgeblich über das Krebsrisiko der Bevölkerung entscheidet.

\section{Haftung}

Mit der Einführung des StrVG ist gleichzeitig eine Einführung von Grenzwerten geplant, die deutlich über dem sogenannten 30-millirem-Konzept der Strahlenschutzverordnung liegen sollen. Dies hat unter anderem zur Folge, daß das Haf- 
tungsrisiko der Bundesrepublik Deutschland gemäß $\ 38$ AtomG drastisch gemindert wird. Würde die Bundesrepublik Deutschland niedrigere Grenzwerte als das umliegende Ausland haben, so müßte sie für Schäden, die vom Ausland ausgehen, eintreten, wenn im betreffenden Ausland ein Schaden nicht in Betracht kommt, weil die Grenzwertschwelle nicht überschritten ist. Haftungsgrundlage wäre dann $\ 38$ AtomG. Durch die Anhebung der Grenzwerte auf ein Niveau, das international gleichliegt, werden die Probleme im Zusammenhang mit $\$ 38$ AtomG, die übrigens schon zu einigen gerichtlichen Verfahren geführt haben, beseitigt.

\section{Auswirkungen des StrVG auf $A K W$}

Wegen der Strahlenbelastung nach Tschernobyl ist das stets zu befolgende $30-$ millirem-Konzept im Normalbetrieb einer atomaren Anlage nicht mehr einzuhalten, da die Werte bereits durch die Vorbelastung überschritten sind. Dies hätte nach der Strahlenschutzverordnung zur Konsequenz, daß neue AKWs in Zukunft nicht mehr genehmigt werden könnten. Überdies wäre auch ein Weiterbetrieb alter AKWs - sieht man einmal von der Bestandsschutzproblematik ab - nicht mehr möglich. Durch die im Zusammenhang mit dem Strahlenschutzvorsorgegesetz beabsichtigte Anpassung im Sinne einer Erhöhung der Grenzwerte an die internationalen Standards wird gewährleistet, daß die Bundesregierung auch in Zukunft die Atomtechnologie anwenden kann.?

\section{Ist ein Radioaktivitätsüberwachungsgesetz erforderlich?}

Ist das Strahlenschutzvorsorgegesetz ein Gesetz, das die Bevölkerung prophylaktisch vor Strahlen schützt? Wohl kaum. Konzeptionell zielt das Gesetz darauf ab, durch Monopolisierung von Informationen, durch situative zentrale Grenzwertfestlegung und -bewertung unter Bezugnahme auf Sachverständige und durch bundeseinheitliche Empfehlungen zu bewirken, daß Ängste der Bevölkerung kanalisiert und kontrolliert werden. Es entspricht insofern einem Typus von Technologiegesetz, das allein die Folgen von Unfällen und Katastrophen reguliert, ohne prophylaktische Bedeutung zu haben.

Es ist erforderlich, die Radioaktivität der Umwelt zu überwachen, und zwar unabhängig von der Frage, ob AKWs sofort, in zehn Jahren oder erst im nächsten Jahrhundert abgeschaltet werden. Denn selbst abgeschaltete AKWs geben radioaktive Strahlen an die Umwelt ab. Im übrigen ist die Langzeitbelastung von niedriger radioaktiver Bestrahlung wissenschaftlich weitgehend unerforscht. Das Erfordernis eines Radioaktivitätsüberwachungsgesetzes gilt natürlich um so mehr, wenn die gegenwärtige Atomenergiepolitik der Bundesrepublik fortgesetzt wird: Allein i985 haben sich in deutschen Reaktoren 239 Störfälle ereignet, wurden 22 Schnellabschaltungen vorgenommen. ${ }^{8}$

Ein Radioaktivitätsüberwachungsgesetz, das eine Alternative zum Strahlenschutzvorsorgegesetz darstellt, muß konzeptionell die Erfahrung verarbeiten, daß die Fortschritte, die im Umweltrecht erzielt worden sind, insbesondere auf die Umweltbewegung zurückzuführen sind: Der Sachverstand der Bürgerinnen und Bürger

\footnotetext{
7 Hierauf hat wiederholt R. Geulen hingewiesen.

8 Aus: «Übersıcht über besondere Vorkommnisse in Kernkraftwerken der Bundesrepublik Deutschland $\alpha$, Bericht der Gesellschaft für Reaktorsicherheit, August 1986.
} 
konnte sich in historisch beispielloser Weise gegen den Sachverstand von Industrie, Politik und Fachwissenschaft durchsetzen. Die vermeintlich irrationale Angst der Bürger erwies sich im Prozeß der politischen Auseinandersetzung als begründet. Deswegen muß ein Radioaktivitätsüberwachungsgesetz bürgerfreundlich sein: Die Meßdaten müssen jedem Mann und jeder Frau zur Verfügung stehen, es darf kein Informationsmonopol geben. Gleichzeitig müssen auch Meßdaten von nicht-staatlichen Stellen in ein Informationssystem integriert werden, da erfahrungsgemäß die Datenerfassung nicht unabhängig ist vom politisch-wissenschaftlichen Standort des Messenden. Bei der Bewertung gewonnener Meßdaten darf es keinerlei Bewertungsmonopol geben. Zur Zeit läßt sich in vielen Fragen wie etwa der Radioaktivitätsbelastung kein wissenschaftlicher Konsens über die Gesundheitsverträglichkeit erzielen. Es bestehen unterschiedliche "Wahrheiten«. Die Bewertung wissenschaftlich kontroverser Positionen muß daher der Bevölkerung überlassen bleiben, alles andere wäre zum Gesetz erhobene Willkür. Letztlich müssen zulässige Obergrenzen und ein Minimierungsgebot gesetzlich festgelegt werden, um zu verhindern, daß Obergrenzen nach aktuellen politischen Opportunitäten festgelegt und sich nach einer neuen Katastrophe nichts weiter ändert außer den zulässigen Obergrenzen.

Ein solches Gesetz, das sich durch Transparenz, Herstellung eines öffentlichen Diskurses, die Bewertung erhobener Daten durch die Bevölkerung und die Festlegung zulässiger Höchstgrenzen einschließlich eines Minimierungsgebotes auszeichnet, ist umweltfreundlich und zugleich demokratisch, im Gegensatz zum Strahlenschutzvorsorgegesetz: Es hat mit Demokratie bestenfalls etwas bis zur Verabschiedung des Gesetzes zu tun. Der Inhalt des Gesetzes stellt eine Enteignung der Gesundheit der Bürger und Bürgerinnen zugunsten des Bundesministers für Umwelt, Naturschutz und Reaktorsicherheit dar.

\section{Joachim Perels Anwaltschaft und demokratische Rechtskultur*}

Die gegenwärtige Rolle der linken Anwaltschaft steht im Spannungsfeld einer machtgestützten Funktionalisierung des Rechts und der - geschichtlich neuen Ausbildung einer demokratischen Gegenkultur auch in der Justiz. Der Stellenwert dieser widersprüchlichen Gesamtlage erschließt sich, wenn man sich die unterschiedlichen Konstellationen von kritischer Anwaltschaft und Justizsystem für den Bereich des Verfassungsrechts, des Strafrechts und des Strafprozeßrechts in der Weimarer Republik, im Dritten Reich und in der frühen Bundesrepublik - zugegebenermaßen kursorisch - vor Augen führt.

\section{Weimarer Republik: Autoritäre Justiz contra linke Anwaltschaft}

In der Weimarer Republik standen liberale und sozialistische Anwaltschaft - als deren herausragende Vertreter etwa Max Alsberg, Max Hirschberg, Kurt Rosenfeld

\footnotetext{
* Referat auf dem 2. Republikanıschen Anwältınnen- und Anwältetag: Zwanzig Jahre *linke * Anwaltschaft - von der APO bis heute -, Berlin 26.-28. 9. 1986. Der folgende Text ist ene überarbeitete Fassung.
} 